\title{
Characterization of Celecoxib-Loaded Solid Lipid Nanoparticles Formulated with Tristearin and Softisan 100
}

\author{
Ehab A Fouad ${ }^{1,2 *}$, Alaa Eldeen B Yassin ${ }^{3,4}$ and Hamdan N Alajami ${ }^{5}$ \\ ${ }^{1}$ Department of Pharmaceutics, Almaarefa Colleges for Science and Technology, P.O Box 71666, Riyadh 11597, Saudi Arabia, \\ ${ }^{2}$ Department of Pharmaceutics, Faculty of Pharmacy, Assiut University 71526, Assiut, Egypt, ${ }^{3}$ Pharmaceutical Sciences \\ Department, College of Pharmacy-3163, King Saud bin Abdulaziz University for Health Sciences, and King Abdullah \\ International Medical Research Center, Ministry of National Guard, Health Affairs, Riyadh 11426, Saudi Arabia, ${ }^{4}$ Department of \\ Pharmaceutics, Faculty of Pharmacy, AlAzhar University, Cairo, Egypt, ${ }^{5}$ Pharmaceutical Care Division, King Faisal Specialist \\ Hospital and Research Centre, PO Box 3354 Riyadh 11211, Saudi Arabia
}

*For correspondence: Email: bahe2004@yahoo.co.uk; Tel: +966 553889130

Received: 22 September 2014

Revised accepted: 1 January 2015

\begin{abstract}
Purpose: To prepare solid lipid nanoparticles employing softisan 100 (SOFTI) or tristearin (TS) as solid lipid carriers for celecoxib (CXB) to overcome its dissolution challenge.

Methods: The solid lipid nanoparticles (SLN) of CXB were prepared by ultrasonic melt-emulsification technique. SLN was characterized using differential scanning calorimetry (DSC), Fourier transform infra spectroscopy (FTIR), as well as for entrapment efficiency, particle size, zeta potential and CXB release. Results: The SLN formulations exhibited high CXB entrapment efficiency (91.6 \% for SOFTI and 94.6 $\%$ for TS) while mean particle size was $181.0 \pm 4.6$ and $346.3 \pm 3.8 \mathrm{~nm}$ for SOFTI and TS, respectively. The DSC thermograms showed the disappearance of CXB peak due to its molecular distribution in the lipid nanoparticles while FTIR spectra revealed physical interaction of CXB with the tested lipids. The tendency of SOFTI to liberate CXB in $24 h$ was higher than that of TS (55.5 \pm 1.07 vs $49.2 \pm 2.94 \%, p<$ 0.05). Drug release was by non-Fickian mechanism.

Conclusion: Formulation of CXB in SLN using TS or SOFTI produces sustained drug release delivery that can overcome the dissolution limitation of the drug and thus, improve its therapeutic efficacy.
\end{abstract}

Keywords: Celecoxib, Solid lipid nanoparticles, Tristearin, Softisan, Dissolution limitation, Sustained drug release

Tropical Journal of Pharmaceutical Research is indexed by Science Citation Index (SciSearch), Scopus, International Pharmaceutical Abstract, Chemical Abstracts, Embase, Index Copernicus, EBSCO, African Index Medicus, JournalSeek, Journal Citation Reports/Science Edition, Directory of Open Access Journals (DOAJ), African Journal Online, Bioline International, Open-J-Gate and Pharmacy Abstracts

\section{INTRODUCTION}

Celecoxib (CXB) is a nonsteroidal antiinflammatory drug used for the treatment of osteoarthritis and rheumatoid arthritis. It is a selective inhibitor for cyclooxygenase-2 (Cox-2), that is expressed during inflammation [1,2]. Hence, CXB does not cause gastropathy or GI bleeding [1]. CXB has a chemopreventive activity toward colon cancer [2], skin cancer induced by UV light [3] and breast cancer [4]. CXB has a half-life of $21 \mathrm{~h}$ with poor oral bioavailability of about $22-40 \%$ from capsule formulations. The poor oral bioavailability of CXB was attributed to its poor solubility and poor dissolution rate [5].

Different techniques have been used to improve drugs bioavailability by increasing their dissolution rate $[6,7]$, the use of controlled release technique for NSAIDs attractive in this regard [8]. For example, the release of CXB from cellulose mixture was extended up to $21 \mathrm{~h}$ in a biphasic fashion, with $50 \%$ released between 75 and $140 \mathrm{~h}$ for ethyl cellulose and 2.8 to $6.5 \mathrm{~h}$ for 
HPMC [9]. Using a pharmacodynamic model, topical nanostructured lipid carrier gel of CXB showed faster onset and prolonged activity up to $24 \mathrm{~h}$ [10]. Solid lipid nanoparticles (SLNs) have been considered as an alternative drug carrier system with several advantages, including enhanced physical stability, dual loading ability for lipophilic and hydrophilic drugs, improved physical stability, low cost, and high feasibility for large scale manufacturing. The most commonly used lipids are triglyceride esters of saturated fatty acids viz tristearin, tripalmitin (TS) trimyristin. Hydrogenated oils such as cottonseed oil, palm oil, castor oil and soybean oil [11]. The prepared SLN can be administered orally, intravenously or topically $[11,12]$.

The objective of the present study was to investigate the use of nanolipid technology for controlling the release of CXB using TS and SOFTI as lipid carriers to overcome CXB dissolution problem.

\section{EXPERIMENTAL}

\section{Materials}

Tristearin (TS) was purchased from Sasol Germany GmbH (Witten, Germany), softisan 100 (SOFTI, saturated glycerides) purchased from CREMER OLEO GmbH \& Co. KG (Hamburg, Deutschland), celecoxib (CXB) was kindly donated by Searle (Augusta, GA, USA). Sodium deoxycholate from Sigma-Aldrich Chemical Company (St. Louis, MO, USA), tween 80 was purchased from BDH, Prolabo, England, All other chemicals were of analytical grade.

\section{Preparation of CXB-loaded SLN}

Accurately weighed $450 \mathrm{mg}$ of lipid (TS or SOFTI) was melted at $10{ }^{\circ} \mathrm{C}$ over their melting points, then $50 \mathrm{mg}$ of CXB was dissolved in the melted lipid (lipid phase). At the same time, $4.5 \mathrm{~g}$ of an aqueous solution containing $1 \%$ tween 80 and $0.5 \%$ sodium deoxycholate (aqueous phase) was heated to the same temperature. The two phases were mixed together and sonicated using probe sonicator for $3 \mathrm{~min}$ at 40 $\%$ voltage efficiency. The formed emulsion was dispersed in chilled $5 \%$ glucose solution (cryoprotector) with vigorous stirring for $3 \mathrm{~min}$ using magnetic stirrer. After that, the formed SLN were stabilized by lyophilization over a period of 72 h using a freeze-drier (Alpha 1-4 LD-2, Martin Christ, Osterode, Germany) under the following conditions: temperature $=-59{ }^{\circ} \mathrm{C}$, vacuum $=$ 0.090 mbar [13].

\section{Evaluation of particle size and polydispersity index}

The $z p$, mean particle size and polydispersity index of SLN for size distribution was measured using photon correlation spectroscopy incorporating a 90 Plus particle size analyzer and a zeta-sizer software (Brookhaven Instruments Corporation Holtsville, New York, USA). The SLN formulations were diluted in a ratio of 1: $1000 \mathrm{v} / \mathrm{v}$ with distilled water before analysis. The angle of detection was $90^{\circ}$ at $25^{\circ} \mathrm{C}$. The polydispersity of SLN formulations were detected in the same condition.

\section{Differential scanning calormetry (DSC)}

Differential scanning calorimeter Shimadzu DSC60 equipped with an intercooler (Shimadzu Corporation, Koyoto, Japan) was utilized for recording thermograms of pure drug, lipids, blank SLN, and drug-loaded SLN. The temperature and enthalpy scale was calibrated using Indium/zinc standard. The samples were hermetically sealed in aluminum pans and heated over a temperature range of $25-200{ }^{\circ} \mathrm{C}$ under nitrogen gas purged at a flow rate of 50 $\mathrm{ml} / \mathrm{min}$. A constant flow rate of $10^{\circ} \mathrm{C} / \mathrm{min}$ was employed.

\section{Fourier transforms infrared spectroscopy (FTIR)}

Fourier transform infrared spectrum (FTIR) was recorded on Perkin Elmer FTIR instrument (Perkin Elmer, Waltham, Massachusetts, USA). A blank $\mathrm{KBr}$ pellet was used as a background. After the correction of the baseline, samples were scanned at a wave number from $4000-400$ $\mathrm{cm}^{-1}$ and a resolution of $1.0 \mathrm{~cm}^{-1}$.

\section{Measurement of drug entrapment efficiency}

The entrapment efficiency (EE) of CXB in SLNs formulations were determined by centrifugation of the colloidal samples at $50,000 \mathrm{rpm}$ at $4{ }^{\circ} \mathrm{C}$ for $30 \mathrm{~min}$. HPLC method was used to determine the non-entrapped $\mathrm{CXB}$ in the supernatant. EE (\%) of CXB was computed as in Eq 1 [13].

$E E(\%)=\{(W i-W f) / W i\} 100$

where Wi is the initial weight used and Wf is the amount of the free drug in the medium.

\section{In vitro release profile study}

Certain weight from each formula equivalent to 1 mg CXB was immersed in dialysis tube with $1 \mathrm{~mL}$ of phosphate buffer $\mathrm{PH}$ 6.8. The dialysis tube 
was made by seamless regenerated cellulose with a typical molecular weight cut-off of 12,000 to 14,000 Dalton. The dialysis tubes were sealed by special clips and immersed in a beaker containing $40 \mathrm{ml}$ phosphate buffer $\mathrm{pH}$ 6.8. Thereafter, beakers were immersed in a water bath at $37{ }^{\circ} \mathrm{C}$ and stirred at $80 \mathrm{rpm}$ speed. Aliquots of $10 \mathrm{ml}$ were removed at specified time intervals and replaced with an equal volume of fresh buffer, in order to maintain sink condition. Finally, the drug released was determined using HPLC.

\section{HPLC Method for CXB analysis}

A modified simple sensitive HPLC method with UV detection as previously described [14] was applied. Briefly, the HPLC system consisted of a Waters Model 1515 HPLC pump, a Waters auto sampler, Model 717 plus and a Waters 2487 dual absorbance UV detector (Waters Inc., Bedford, MA, USA) governed by a microcomputer running Empower $\circledR$ software (version 1154). The detector was set at $260 \mathrm{~nm}$ to detect CXB. The separation system was C18 analytical, $\mu$ Bondapack column $(150 \mathrm{~mm}$ length $\times 4.6 \mathrm{~mm}$ inner diameter. $10 \mu \mathrm{m}$ particle size). The mobile phase consisted of acetonitrile: water (60: 40 $(\mathrm{v} / \mathrm{v})$ ) and isocratic technique was used. Methanolic solution of celecoxib was used for preparing the standard calibration curve.

\section{Release kinetic analysis}

The fraction of total CXB amount released (Mt / $M \infty$ ) was fitted with time $t$ according to the Higushi diffusion model as well as the Peppas and Korsemayer model using the following equations:

Mt $/ \mathrm{M}_{\infty}=\mathrm{k}_{\mathrm{h}} \mathrm{t}^{1 / 2}$ (Higuchi model) where $k_{h}$ is the Higuchi diffusion rate constant.

Mt / M $\infty=k_{p} t^{n}$ (Peppas-Korsemayer model) .. (3) where $k_{p}$ is the release rate constant at the elapsed time $\mathrm{t}, \mathrm{n}$ is a constant, where the value of $n \leq 0.45$ indicates Fickian diffusion, $0.45 \leq n \leq$ 0.89 indicates non-Fickian (anomalous) diffusion, $\mathrm{n}=0.89$ indicates case-Il transport (erosion control and zero-order kinetics), and $n \geq 0.89$ indicates Super Case II transport [15].

The MULTI computer program was utilized for equations fitting with little modifications. In addition to, the examination of the predicted curve fitted to the data, the sum of the squared residuals (SSR) and Akaike's information criterion (AIC) was employed as the main criteria for judging the best fitting [16]. AIC can be calculated using Eq 4 while SSR was obtained as in Eq 5.

$A I C=n[\ln (S S R)]+2 p$

$\operatorname{SSR}=\operatorname{Wij}\left(C_{i, j}-\int\left(t_{j}, P\right)\right) 2$

where $\mathrm{n}$ is the number of experimental points, and $p$, is the number of parameters to be estimated [15].

\section{Statistical analysis}

Data analysis was performed using the Statistical Package for Social Sciences (SPSS ver. 19.0). One-way analysis of variance (ANOVA) was applied to determine statistical differences, and differences were considered significant at $p<$ 0.05 .

\section{RESULTS}

\section{Physical parameters}

The mean physical parameters of the prepared SLN containing CXB showed some differences between SOFTI and TS, The recorded mean particle size was significantly different $(p<0.05)$ with $181.0 \pm 4.6$ and $346.3 \pm 3.8 \mathrm{~nm}$ for SOFTI and TS, respectively. Polydispersity index was $0.325 \pm 0.013$ which was significantly lower than that of TS $(0.379 \pm 0.002),(p<0.05)$ appears acceptable for such nanoparticles.

Zeta potential is the factor that reflects the colloidal dispersion stability of the prepared SLN. It was found that zeta potentials above $30 \mathrm{mV}$ were required for full electrostatic stabilization [17]. The measured zeta potential values of CXB loaded SLN preparations were significantly different $(p<0.05),-45.7 \pm 3.1$ and $-64.0 \pm 4.4$ for SOFTI and TS, respectively. The entrapment efficiency (EE, \%) for CXB into SLN was measured. SLN prepared using SOFTI showed $91.6 \%$ EE, while that of TS was 94.6.

Fig 1 shows the DSC thermogram of CXB, lipids and SLN, while Table 1 denotes peak temperatures and enthalpy change of the prepared SLN. In pure CXB thermogram a single endothermic melting peak of the crystalline form present at $164.74{ }^{\circ} \mathrm{C}$. Each of the pure lipids showed a specific melting endothermic peak that was sharp in case of TS at $75.86{ }^{\circ} \mathrm{C}$, but was not sharp at $34.75^{\circ} \mathrm{C}$ in case of SOFTI. This finding may be due to the presence of different fatty acid esters (C10-C18) in SOFTI composition. Dextrose also has a sharp melting peak at 
$154.84{ }^{\circ} \mathrm{C}$. SOFTI-SLN thermogram showed a lipid endothermic peak little shifted to a lower temperature at $33.7{ }^{\circ} \mathrm{C}$; however, the $\mathrm{CXB}$ completely disappeared. On the other hand, TSSLN thermogram showed a lipid endothermic peak shifted to $70.51{ }^{\circ} \mathrm{C}$ with no CXB peak.

\section{FTIR study}

Figure 2 presents the FTIR spectrum of CXB, lipids and SLN. It is clear that the bands at
3339.86 and $3233.76 \mathrm{~cm}^{-1}$ for CXB (due to $\mathrm{NH}_{2}$ ) completely disappeared in SLN with the formation of wide broad band at $3398.15 \mathrm{~cm}^{-1}$ in case of SOFTI-SLN and 3421.83 in TS-SLN. However, there aren't any peaks existing in this range for pure lipids.

The in vitro drug release study was conducted using membrane diffusion techniques. Figure 3 shows the release profile of CXB from SOFTI-

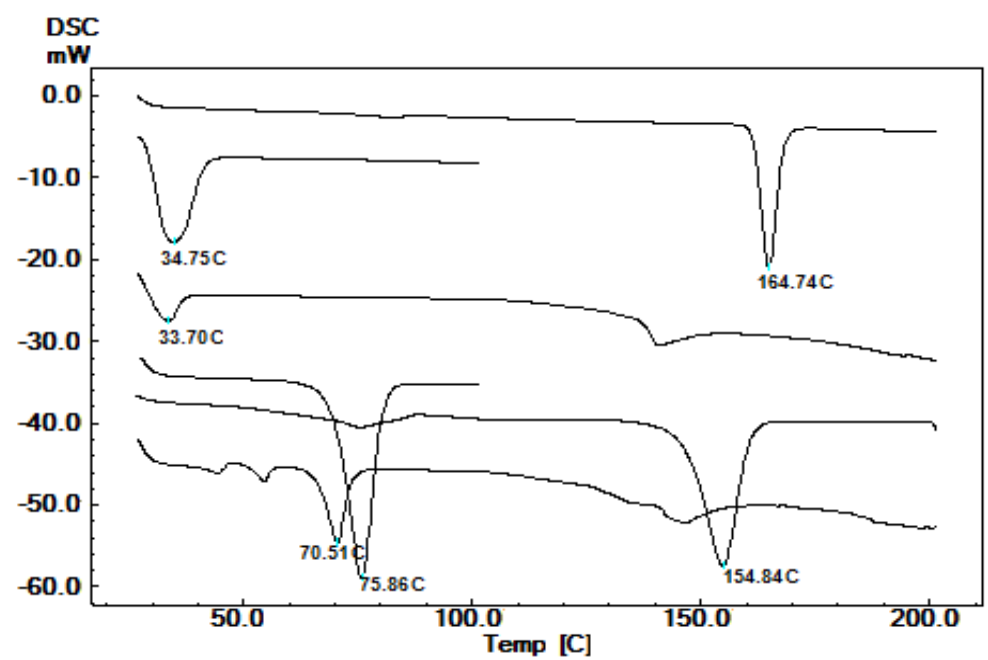

Figure 1: DSC thermogram of CXB, lipids, SLN and dextrose (DEX)

Table 1: DSC peak temperature and enthalpy change of lipids and SLN

\begin{tabular}{lcccc}
\hline Ingredient & Peak (1) temp $\left({ }^{\circ} \mathbf{C}\right)$ & $\Delta \mathbf{H}(\mathbf{1}) \mathbf{j} / \mathbf{g}$ & $\begin{array}{c}\text { Peak (2) temp } \\
\left({ }^{\circ} \mathbf{C}\right)\end{array}$ & $\Delta \mathbf{H}(\mathbf{2}) \mathbf{j} / \mathbf{g}$ \\
\hline Celecoxib & 164.74 & -110.99 & -- & --- \\
Tristearin & 75.86 & -202.27 & -- & --- \\
Tristearin SLN & 70.51 & -70.59 & 146.51 & -9.4 \\
Softisan 100 & 34.75 & -145.15 & -- & --- \\
Softisan 100 SLN & 33.70 & -25.37 & 140.84 & -23.77 \\
\hline
\end{tabular}

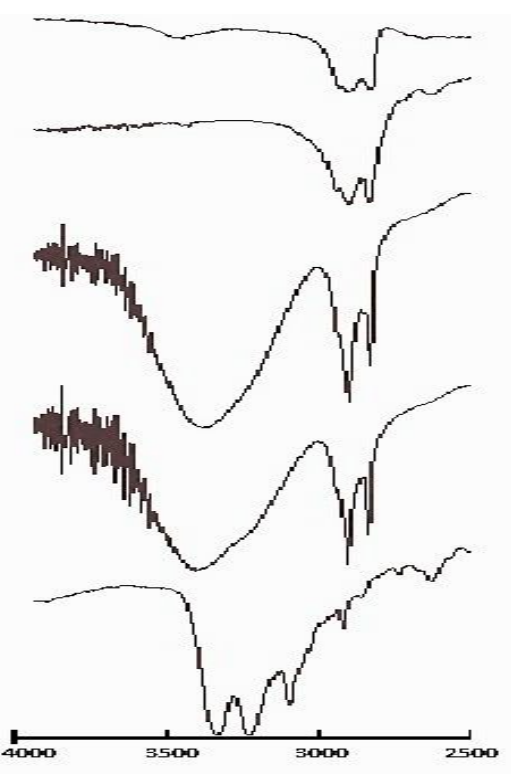

Figure 2: FTIR spectra for CXB, lipids and SLN

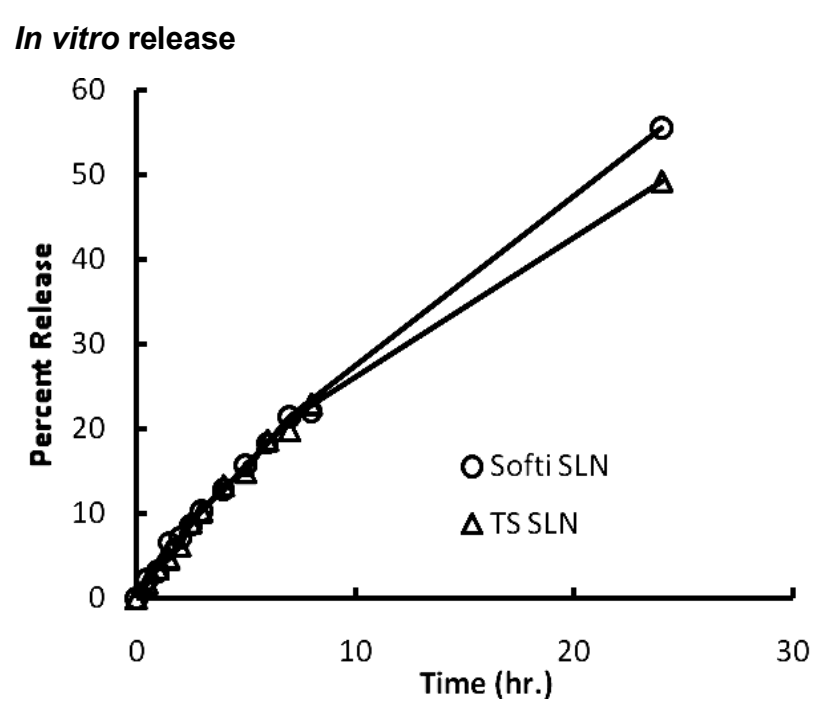

Figure 3 : Release profile of CXB from SLN

Trop J Pharm Res, February 2015; 14(2): 208 
Table 2: Release-fitting parameters of CXB from SLN

\begin{tabular}{lccccccc}
\hline \multirow{2}{*}{ Formulation } & \multicolumn{3}{c}{ Higuchi diffusion model } & \multicolumn{3}{c}{ Peppas-Korsemayer model } \\
\cline { 2 - 7 } & $\boldsymbol{K}$ & $\boldsymbol{A I C}$ & $\boldsymbol{S S R}$ & $\boldsymbol{A I C}$ & $\boldsymbol{S S R}$ & $\boldsymbol{K}$ & $\boldsymbol{n}$ \\
\hline SOFTI & 0.125 & -38.47 & $2.52 \times 10^{-2}$ & -62.20 & $2.43 \times 10^{-3}$ & 0.069 & 0.869 \\
TS & 0.139 & -32.43 & $3.19 \times 10^{-2}$ & -49.49 & $4.75 \times 10^{-3}$ & 0.075 & 0.882 \\
\hline
\end{tabular}

SLN as well as TS-SLN. The release of CXB from SLN was slow from the two formulations and did not show any significant difference $(p>$ 0.05 ) up to ten hour. After that, there was a significant increase $(p<0.05)$ in CXB release from SOFTI than that from TS $(55.5 \pm 1.1$ vs 49.2 \pm 2.9 ) after $24 \mathrm{~h}$. In addition, the release profile existed as more than one phase. Table 2 depicts the calculated release parameters according to Higuchi diffusion as well as Peppas-Korsemayer models.

\section{DISCUSSION}

The measured physical parameters revealed that the prepared SLN has a good stability toward agglomeration. Also, these results reflect the effect of lipid structure on the prepared SLN, where TS is a mono acid (C18) triglyceride; however, SOFTI is a mixture of glycerides (C10$\mathrm{C18}$ ). The values of \% EE reflect the solubility and affinity of $C X B$ to the used lipids; also, the lipophilicity of $\mathrm{CXB}$ with the higher amount of lipids may play a role toward increasing the entrapment efficiency. These result are in agreement with Kim et al [18] who got entrapment $>75 \%$ for verapamil when its ratio to lipid was 1:5 and 1:10.

In the present study CXB dissolved completely in the melted lipid phase and the lipid phase was completely clear, so, CXB was molecularly dispersed as solute in the oil phase as solvent. The possibility of interaction between the amino group of $\mathrm{CXB}\left(\mathrm{NH}_{2}\right)$ and the carbonyl group $(\mathrm{C}=\mathrm{O})$ of the glyceryl ester in the lipid is probable, especially in the presence of surfactant. On the other hand there is a possibility for hydrophobic interaction between $\mathrm{CXB}$ and the hydrocarbon chain of the esterified fatty acids in the lipids. Upon emulsification CXB partitioned between the lipid and the aqueous phase. Since $C X B$ is a hydrophobic drug, it showed a great affinity towards lipids hence achieving EE up to $94.624 \%$ in case of TS and $91.554 \%$ in case of SOFTI. After solidification the DSC endothermic peak of CXB completely disappeared. This finding may be attributed to the embedding and molecular distribution of CXB into the lipid where no aggregation of $\mathrm{CXB}$ molecules together which means that upon heating $\mathrm{CXB}$ molecules will appear as molecularly dissolved in the melted lipid with the gradual heating in the DSC. The DSC peaks of the lipids in the SLN were shifted towards lower temperature with lower heat which may be due to lipid polymorphism and/or the interaction with CXB. The FTIR data confirmed the DSC finding through the disappearance of the $\mathrm{NH} 2$ bands at 3339.86 and $3233.76 \mathrm{~cm}^{-1}$ with the appearance of a wide broad band at 3398.15 for SOFTI-SLN and 3421.83 for TS-SLN.

Upon emulsification the surfactant mixture of tween 80 and sodium deoxycholate formed a mixed micelle solution that stabilized the emulsion of lipid into water forming a thin micellar coat around the solid lipid nanoparticles. Accordingly the affinity of $\mathrm{CXB}$ to the lipid phase and the micellar coat will affect the partition of the drug and its entrapment efficiency during the formulation of the SLN. Also, this embedding will affect the drug release and its characteristics. The early appearance of the melting peak can also be attributed to the small particle size of SLN that melt faster at lower temperature as found by other authors [19].

The release behavior of CXB may be attributed to the complexity of the SLN systems. It is clear that more than one release mechanisms are going to occur. Those mechanisms may take place simultaneous or to pursue one another. The distribution of the drug in SLN usually arises in two positions. The first is the molecularly dissolved in the lipid particles, while the second is on the surface of the particles that consisted of mixed micellar coat of tween 80 and sodium deoxycholate (inside the micelles and adsorbed on the micellar coat). As the drug dissolved completely in the lipid during SLN preparation, then upon mixing with the micellar solution the drug is going to partition between the two phases. In the aqueous phase the drug equilibrates between micellar core and free water. At the end of freeze drying the amount of drug in the free water is adsorbed on the micellar coat. During dissolution/release, the amount of drug adsorbed on the surface will diffuse out first together with that released from the micellar coat. At the same time water penetrates the lipid matrix through surface emulsification of the particles causing a change in its consistency followed by drug release that is controlled by partitioning between the lipid phase and the aqueous micellar phase in the dialysis bag. The drug release from the present system will depend 
on partitioning [20]. Table 2 depicts the calculated release parameters, according to the $\mathrm{n}$ values, the release appear as a non fickian mechanism.

\section{CONCLUSION}

CXB embedded in SLN nanoparticles, prepared using TS and SOFTI as lipid carriers, show suitable particle size and polydispersity index. CXB release from SLN occurs slowly in a nonFickian diffusion pattern. CXB appears to be distributed molecularly in the lipid carrier with probable physical interaction with the lipids leading to slow release. Thus, SLN appears promising a sustained delivery system for CXB to overcome its poor dissolution.

\section{ACKNOWLEDGEMENT}

The authors acknowledge the generous financial support from King Abdul Aziz City for Science and Technology (KACST) (A-T-34-71), Saudi Arabia.

\section{REFERENCES}

1. Fort J. Celecoxib, a COX-2 specific inhibitor: the clinical data. Am J Orthop 1999; 28(3): 13-18.

2. Kawamori $T$, Rrao CV, Seibert $K$, Reddy BS. Chemopreventive activity of celecoxib, a specific cyclooxygenase-2 inhibitor, against colon carcinogenesis. Cancer Res 1998; 58(3): 409-412.

3. Fisher SM, Lo HH, Gordon GB, Seibert K, Kellof G, Lubet $R A$, Conti CJ. Chemopreventive activity of celecoxib, a specific cyclooxygenase-2 inhibitor, and indomethacin against ultraviolet light-induced skin carcinogenesis. Mol Carcinog 1999; 25(4): 231-240.

4. Harris RE, Alshafie GA, Asbou-issa $H$, Seibert $K$. Chemoprevention of Breast Cancer in Rats by Celecoxib, a Cyclooxygenase 2 Inhibitor. Cancer Res 2000; 60(8): 2101-2103.

5. Pualson SK, Vaughan MB, Jessen SM, Lawal Y, Gresk CJ, Yan B, Maziasz TJ, Cook CS, Karim A. Pharmacokinetics of celecoxib after oral administration in dogs and humans: effect of food and site of absorption. J Pharmacol Exp Ther 2001; 297 (2), 638-645.

6. Leuner C, Dressman J. Improving drug solubility for oral delivery using solid dispersions. Eur J Pharm Biopharm 2000; 50: 47-60.

7. Hancock BC, Zografi G. Characteristics and significance of the amorphous state in pharmaceutical systems. J Pharm Sci 1997; 86: 1-12.
8. Falco G, Borghi C, Berti F. Non-steroidal antiinflammatory drug therapy: advantages and disadvantages of long half-life drugs versus slowrelease formulations. Int J Clin Pharmacol Res 1986; 6: 475-480.

9. Chandran S, Ravi P, Saha RN. Development and In Vitro evaluation of oral controlled release formulations of celecoxib using optimization technique. Yakugaku Zasshi 2006; 126(7): 505-514.

10. Joshi M, Patravale V, Nanostructured lipid carrier (NLC) based gel of celecoxib. Int J Pharm 2008; 346: 124 132.

11. MacGregor KJ, Embleton JK, Lacy JE, Perry EA, Solomon LJ, Seager H, Poulton CW. Influence of lipolysis on drug absorption from the gastro-intestinal tract. Adv Drug Deliver Rev 1997; 25: 33-46.

12. Dingler A, Runge S, Muller RH. SLN (Solid Lipid Nanoparticles) as drug carrier for an IV administration of poorly water soluble drugs. Eur J Pharm Sci 1996; 4 (Supplement 1): 132-132.

13. Yassin AEB, Anwer MK, Mowafy HA, El-Bagory IM, Bayomi MA, Alsarra IA. Optimization of 5-fluorouracil solid-lipid nanoparticles: a preliminary study to treat colon cancer. Int J Med Sci 2010; 7: 398-408.

14. Jalalizadeh $H$, Amini $M$, Ziaee V, Safa A, Farsam $H$, Shafiee A. Determination of celecoxib in human plasma by high-performance liquid chromatography. J Pharm Biomed Anal 2004; 35, 665-670.

15. Bommareddy GS, Paker-Leggs S, Saripella KK, Neau $S H$, Extruded and spheronized beads containing Carbopo/® 974P to deliver nonelectrolytes and salts of weakly basic drugs. Int J Pharm 2006; 321: 62-71.

16. Yamaoka K, Nakagawa T, Uno T. Application of Akaike's Information Criterion (AIC) in the Evaluation of Linear Pharmacokinetic Equations. J Pharmacokinet Biopharm. 1978; 6 (2): 165-175.

17. Thatipamula RP, Palem CR, Gannu R, Mudragada S, Yamsani MR. Formulation and in vitro characterization of domperidone loaded solid lipid nanoparticles and nanostructured lipid carrier. Daru 2011; 19(1): 23-32.

18. Kim BD Na K, Choi HK. Preparation and characterization of solid lipid Nanoparticles (SLN) made of cacao butter and curdlan. Eur J Pharm Sci 2005; 24: 199205.

19. Reddy LH, Murthy RS. Etoposide-loaded nanoparticles made from glyceride lipids: formulation, characterization, in vitro drug release, and stability evaluation. AAPS PharmSciTech 2005; 6(2): E158E166.

20. Le Verger ML, Fluckiger L, Kim Y, Hoffman M, Maincent $P$. Preparation and characterization of nanoparticles containing antihypertensive agent. Eur J Pharm Biopharm 1998; 46: 137-143. 